\title{
Téoros
}

Revue de recherche en tourisme

\section{Tourisme humanitaire}

Au nom des droits humains et du bien-être pour tous

\section{Julie Laplante}

Volume 24, numéro 3, automne 2005

Tourisme et santé, quelques perspectives

URI : https://id.erudit.org/iderudit/1071078ar

DOI : https://doi.org/10.7202/1071078ar

Aller au sommaire du numéro

Éditeur(s)

Université du Québec à Montréal

ISSN

0712-8657 (imprimé)

1923-2705 (numérique)

Découvrir la revue

\section{Citer cet article}

Laplante, J. (2005). Tourisme humanitaire : au nom des droits humains et du bien-être pour tous. Téoros, 24(3), 30-36. https://doi.org/10.7202/1071078ar d'utilisation que vous pouvez consulter en ligne.

https://apropos.erudit.org/fr/usagers/politique-dutilisation/ 


\section{Tourisme humanitaire \\ Au nom des droits humains et du bien-être pour tous}

\section{Julie Laplante}

La notion de tourisme évoque un élément de loisir et la notion de l'humanitaire un élément de dévouement. Comment ces deux notions peuvent-elles se concilier, se métisser et quel en sont les effets sur le développement? Voilà la question à laquelle nous allons ici tenter de répondre.

Le «droit au plaisir » serait une conquête du XXe siècle (Laplante 1999 : 20). On peut aujourd'hui parler d'une industrie touristique qui doit peut-être son effervescence à l'institutionnalisation du tourisme social ${ }^{1}$ " qui est porteur d'une vision humaniste pouvant répondre aux attentes d'une grande partie des voyageurs et des vacanciers " (Bélanger,1999: 53). Deux pôles du tourisme se dessinent déjà dans ce portrait : le pôle du droit au loisir pour soi et celui du droit social au loisir pour soi et pour les autres. Le second pôle s'inscrit dans les visées humanitaires, mais sa «normativité » aura peut-être l'effet de rendre le premier pôle qui s'y joindra plus extrême.

Une première vague de tourisme peut se répartir en tourisme de masse organisé ou individuel, d'exploration, de tourisme errant (drifter) ; un continuum pouvant indiquer le degré de familiarité-nouveauté recherché, l'errance maximisant la nouveauté, le tourisme de masse organisé la familiarité (Cohen, 2004 : 39). La typologisation peut encore se faire en termes de tourisme de proximité, de tourisme expérimental (Urbain, 1994), de tourisme ethnique (Smith, 1989 ; Picard et Wood, 1997 ; Chambers, 2000 : 100 ) et même de tourisme culturel, de nomadisme d'agrément, de tourisme extrême ou d'expérience touristique originale (Laplante, 1999), toutes des formes de tourisme à la quête de nouvelles expériences et libertés, selon diverses intensités d'exotisme, d'authenticité, de dépaysement, de familiarité et de nouveauté, de rite et de risque. L'ampleur de ces formes de tourisme en fait un phénomène de masse plutôt que des exceptions. Elles visent d'abord et avant tout le loisir, le plaisir et le bien-être personnel, éphémère ou durable, du voyageur.

Une seconde vague de tourisme joint à l'objectif du loisir, du bien-être personnel, une préoccupation pour le bien-être de l'Autre (ou du résidant du pays-hôte), optant pour l'objectif d'un bien-être pour tous. Tourisme mondial, équitable, responsable et durable, tourisme compétitif, qualitatif et éthique, tourisme réfléchi et attentif à la culture de l'Autre (Unesco, 2005), écotourisme, tourisme «faiseur de paix » (Cazes, 1999) : voilà de nouvelles tendances touristiques que semble vouloir prendre le droit au plaisir au $X X I^{e}$ siècle.

En 1995, l'Organisation mondiale du tourisme (OMT) et l'Organisation des Nations unies pour l'éducation, la science et la culture (Unesco) guident l'établissement d'une chartre pour le tourisme durable. En 1998, un entrepreneur égyptien, Moustapha El Gindy, lance la campagne Tourism Against Misery. Un Code mondial éthique du tourisme est adopté en 1999 à Santiago de Chili par les ONG (organisations non gouvernementales) et les associations concernées, notamment par le TEN (Tourism European Network) et sa branche française, l'association "Transverses " ${ }^{2}$. Le droit humain que défend ce code repose sur la liberté des déplacements touristiques : "la possibilité d'accéder, directement et personnellement, à la découverte des richesses de la planète constitue un droit également ouvert à tous les habitants du monde ${ }^{3}$.
La communauté internationale, les ONG et les associations diverses jouent un rôle moralisateur important dans les manières de rendre ce droit opérationnel ; essentiellement à savoir comment effectuer les transferts de connaissances techniques devant mener à ces libertés pour tous. Le "droit au tourisme " s'inscrit à ce titre pleinement dans les visées de l'aide humanitaire, du respect des droits de l'homme et des libertés fondamentales et nous permet d'oser parler de « tourisme humanitaire » comme faisant parti des tendances touristiques contemporaines de cette seconde vague.

Le tourisme humanitaire se différencie de l'aide humanitaire en tant que telle, qui constitue un travail professionnel ardu ; le tourisme humanitaire réfère aux formes de tourisme qui gravitent autour de l'aide humanitaire. Qu'il s'agisse de participer à la construction d'une clinique médicale auprès des autochtones des hauts plateaux boliviens pendant quelques semaines, de consacrer une année de sa retraite à partager ses connaissances techniques avec des homologues des pays en développement, d'une escale de quelque mois, au cours d'un voyage autour du monde, au sein d'un projet de Médecins Sans Frontières subvenant aux besoins qui découlent d'une inondation survenue au Mozambique... plusieurs destinations touristiques sont aujourd'hui choisies en fonction des soins et de l'aide humanitaire que l'on croit pouvoir apporter à des communautés ou à des groupes de population en situation de vulnérabilité. II s'agit essentiellement d'un tourisme éthique, généralement éphémère pour le volontaire, mais se voulant durable pour les populations ciblées. L'expérience touristique originale (Laplante, 1999) qui y est recherchée est fondamentale: être ailleurs, être utile, interagir de manière constructive avec des personnes de cultures variées dans des situations humaines intenses et souvent bouleversantes. Elle attire des professionnels, mais aussi nombre de touristes er- 
rants, ou drifters, qui se greffent sporadiquement à l'aide humanitaire. Cette expérience touristique originale intense s'estompe avec le temps ; soit elle est maintenue par de constants déplacements, soit elle est apaisée par une quête parallèle de diverses formes de tourisme extrême qui gravitent autour de l'aide humanitaire.

Le présent article propose d'abord un parallèle entre la montée de l'humanitaire et la montée des nouvelles tendances de «tourisme réfléchi et attentif à la culture de l'Autre" (Unesco, 2005). Un regard sur une organisation de santé internationale, Médecins Sans Frontières, sert par la suite à comprendre en quoi peut consister le « tourisme humanitaire». La dynamique du tourisme contemporain que cette "nouvelle " forme de tourisme révèle est peut-être celle d'un nouveau rapport à l'Autre, d'un nouvel équilibre entre de nouveaux interdits collectifs et d'anciennes libertés individuelles, entre d'anciens interdits collectifs et de nouvelles libertés individuelles. Nous en arriverons à constater que les relations de pouvoir maintenues à travers les dynamiques de transfert de connaissances techniques humanitaires et touristiques constituent, il va sans dire, des barrières au dialogue entre les cultures et les civilisations, des barrières qui bloquent peut-être la possibilité de la réalisation de ce "bien-être » pour tous.

\section{La montée de l'humanitaire ${ }^{4}$}

La philanthropie de l'aide humanitaire prône la santé pour tous, soit le droit pour tous à un état de complet bien-être physique, mental et social (préambule à la Constitution de l'Organisation mondiale de la santé depuis 1946). Le tourisme se veut et se fait aujourd'hui un moyen pour atteindre cet objectif de bien-être pour tous. Il est depuis longtemps associé à l'amélioration du bien-être personnel. Bien des siècles passés, il a été associé au bien-être spirituel par la voie des pèlerinages. Dès la moitié du XIXe siècle, des bénéfices en termes de santé physique ont été aussi associés au tourisme de loisir (Chambers, 2000: 16). Les bénéfices d'abord perçus en termes d'amélioration du bien-être personnel ont ensuite été regardés en termes sociaux d'amélioration de la productivité dans un système industriel capitaliste devenant de plus en plus compétitif. Mais le tourisme visant le bien-être de l'Autre en même temps que le sien propre semble avoir pris son ampleur avec le récent boom du marché humanitaire.

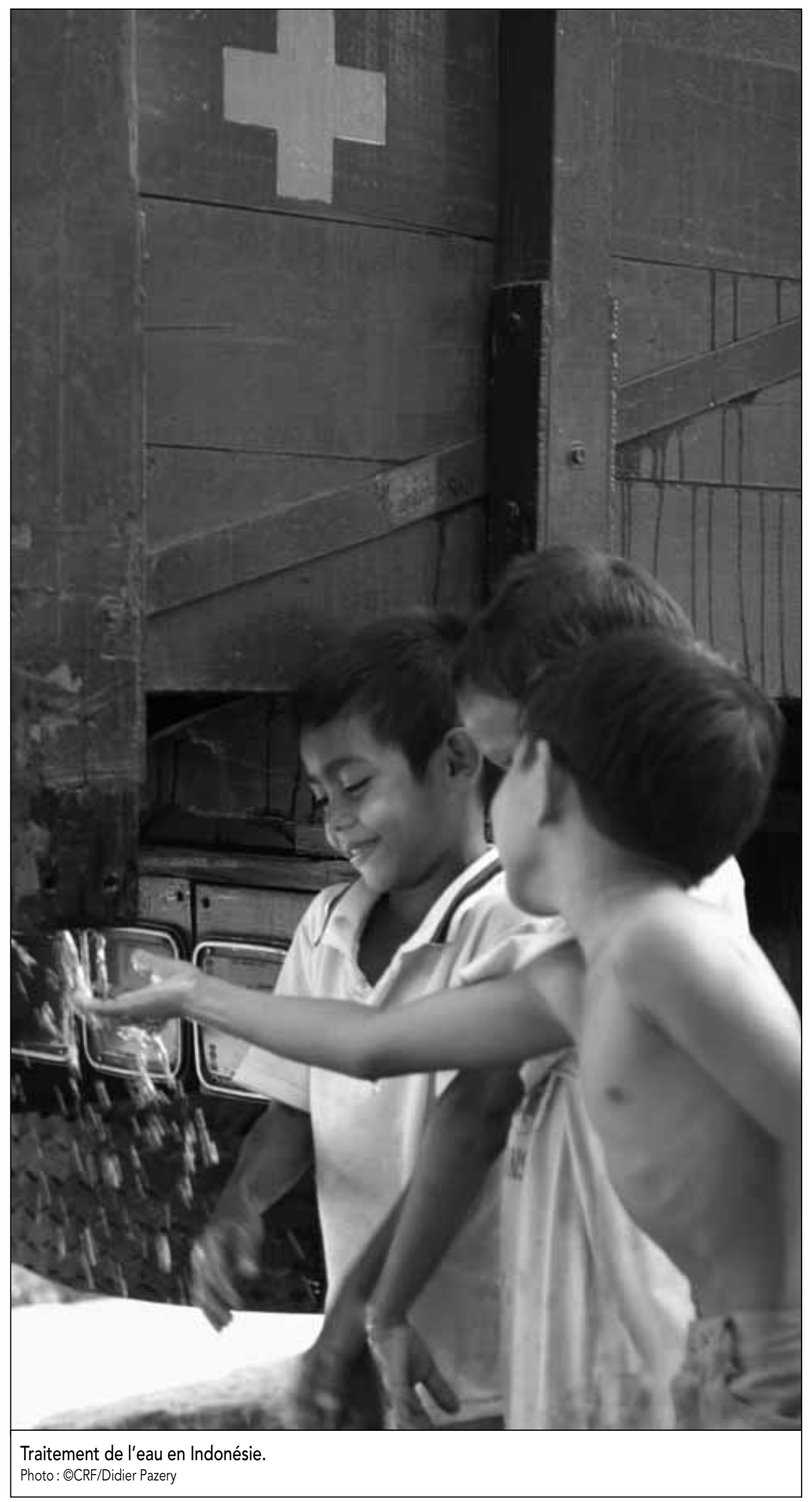




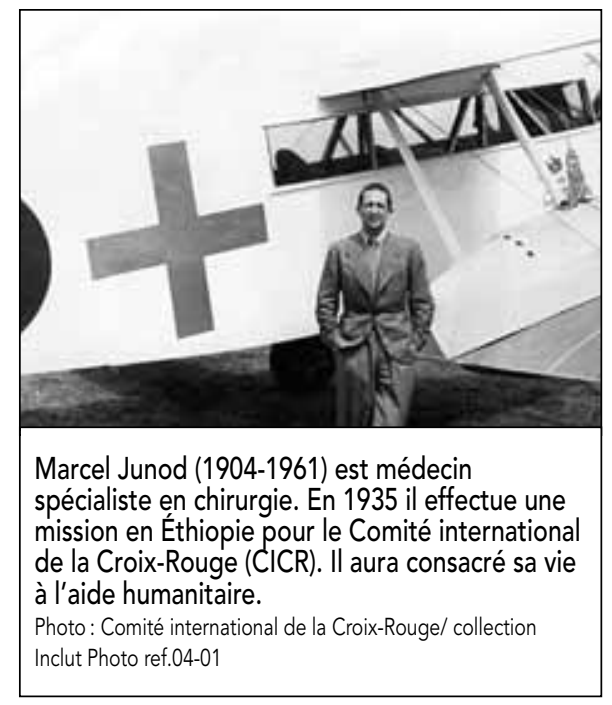

La Croix-Rouge (en Suisse, en 1863) est à l'origine de la création d'une première génération d'ONG modernes dans son combat pour l'universalisation des droits humains et pour l'organisation des secours d'urgence. La notion de droit international humanitaire se développe alors ; toutefois, c'est surtout la Seconde Guerre mondiale et la situation de l'après-guerre qui déclenchent la prolifération d'organisations qui s'efforcent d'apporter d'abord des secours, puis une aide à la reconstruction des pays européens dévastés 5 . Les ONG humanitaires présentes sur la scène internationale à la fin du XXe siècle seraient issues des mouvements caritatifs internes qui furent ultérieurement projetés hors des frontières nationales.

Dans les années 1960-1970, le modèle des missionnaires modernes, contrairement au modèle d'évangélisation prôné du XVI au $\mathrm{XVIII}$ e siècle, allie la conversion à des actions essentiellement centrées sur l'éducation et la santé. À cette période, le développement constitue une aventure morale et humaine axée vers le tiers-monde et la solidarité est essentiellement conçue comme obéissant à une obligation morale : "la notion de développement à long terme et de partenariat va de soi, puisque le développement est envisagé comme un processus pédagogique de longue durée, une mise en valeur des hommes d'abord, et non comme une optimisation des ressources économiques " ${ }^{6}$. Les aspects paternalistes et missionnaires sont profondément ancrés dans ces représentations particulières de la solidarité.
Les ONG de développement proprement dites seraient aussi apparues dans les années 1960 et 1970, parallèlement au mouvement d'accession à l'indépendance des peuples colonisés. La lutte contre la famine est une préoccupation prépondérante. De nombreux pays en développement optent pour la révolution dite verte et ses atouts technologiques, l'introduction de technologies agricoles étant perçue comme le seul moyen d'améliorer les conditions de vie des victimes d'un déficit alimentaire. Elle aura des conséquences désastreuses sur l'environnement et augmentera progressivement la dépendance des pays participants à l'égard des multinationales de l'agro-industrie. Ensuite, c'est de biorévolution (Burch et León, 1996) ou de révolution pharmaceutique dont on parle ; l'accès aux biotechnologies, principalement aux médicaments, est présenté comme la solution mondiale aux problèmes de santé (Laplante, 2003). Les pays ciblés ne possédant ni les savoirs, ni les infrastructures nécessaires au soutien à long terme de l'implantation de ces nouvelles technologies font en sorte d'engendrer de nouvelles formes de dépendance. La biorévolution en région habitée par des populations marginalisées se situe dans le prolongement de la déclaration des droits de I'homme, dans une perspective égalitariste: que tous aient accès aux biotechnologies qui circulent sur les marchés mondiaux en fonction des principes de justice sociale. L'action des ONG humanitaires consiste surtout à s'associer physiquement, sur le terrain, aux efforts de développement des nouveaux pays indépendants, en les faisant bénéficier, au besoin, de l'expérience technique de militants volontaires. Le tiers-monde est considéré comme un terreau favorable au déclenchement d'un processus selon lequel les pays du Nord devraient exporter leurs technologies aux pays défavorisés du Sud. Ces ONG atteignent un début de reconnaissance et de notoriété internationale vers la fin des années 1970.

À partir des années 1970, le " sans-frontiérisme " vient rompre complètement avec les fondements théoriques et pratiques des actions des ONG de développement. L'association MSF a été fondée en 1971, après la guerre au Biafra (1967-1970), autour de Bernard Kouchner qui en a forgé les principales caractéristiques. On cherchait alors les moyens de répondre rapidement et efficacement à des urgences de santé publique, tout en maintenant une indépen- dance totale vis-à-vis tous les pouvoirs impliqués, soit les pouvoirs politiques, économiques et religieux. Aujourd'hui, MSF constitue la plus grande organisation privée de secours médical d'urgence au monde après la Croix-Rouge. Le premier objectif de MSF est de soigner, mais aussi de témoigner, par la voix des médias, d'actes jugés inhumains (advocacy). Le "sans-frontiérisme" réalise une alliance entre professionnalisme, capacité technique et puissance médiatique. Les solutions privilégiées pour faire face aux calamités et aux conflits sont d'ordre biomédical et se traduisent le plus souvent en solutions techniques et logistiques. Dans ce contexte, on ne peut plus opposer la notion d'urgence à celle de développement, comme on le faisait en 1985 (Hours, 1998: 111).

Les controverses avec les « développementalistes » à la suite de la fondation de MSF se sont finalement apaisées, mais elles se trouvent maintenant déplacées à un autre niveau avec l'apparition d'un humanitarisme d'État incarné par le même Bernard Kouchner, devenu secrétaire d'État en France, puis ministre de l'Action humanitaire (et de la Santé). Une nouvelle polémique de plus en plus vive s'ensuit, à partir de 1990. MSF, Médecins du Monde (MDM) et d'autres ONG universalistes humanitaires s'opposent soit au principe soit aux modalités de l'intervention des États dans un domaine qui, estiment ces organisations, ne concerne pas les États. Dans sa charte actuelle, les centres MSF revendiquent, "au nom de l'éthique médicale universelle et du droit à l'assistance humanitaire, la liberté pleine et entière de l'exercice de leur fonction "'?

Parallèlement à ce mouvement du "sansfrontiérisme ", fleurit l'associatif du Sud. Le "sans-frontiérisme" du Nord envisage le développement comme une gestion macroéconomique de la planète, alors que le Sud le conçoit plutôt comme la réalisation des aspirations des habitants urbains et ruraux. Les organisations du mouvement associatif du Sud sont avant tout impliquées sur le terrain et plus étroitement liées à des conceptions locales du développement que ne l'étaient les ONG "sans-frontiéristes", mais certaines de ces organisations ont cependant acquis une dimension internationale ${ }^{8}$.

Une dernière source de la cristallisation de l'intervention humanitaire qui émerge aujourd'hui, et qui englobe le développement au 
sens classique et l'urgence, est d'ordre juridique. Elle correspond à une certaine formalisation de l'émergence de l'individu et de la société civile dans les relations internationales, relations traditionnellement centrées sur les États et les organisations interétatiques (Lecherry et Ryfman, 1993 : 22). Ce sont les dispositions de l'article 71 de la Charte des Nations unies qui, à propos du rôle du Conseil économique et social (ECOSOC) de l'organisation mondiale, ont déterminé pour la première fois un cadre juridique précis régissant les relations entre le système de l'ONU et le mouvement associatif, première étape de la reconnaissance, par la communauté internationale, d'organismes qui ne doivent de ce fait, en principe, ni leur création, ni leur financement aux États. Une ONG devient ainsi toute organisation internationale qui n'est pas créée par des accords intergouvernementaux, mais qui est plutôt issue d'initiatives privées.

Les ONG des années 1990 imposent le concept d'aide humanitaire en remplacement de celui de développement. Elles manifestent une volonté d'ingérence, se proposent comme une nécessité morale et humanitaire au nom de l'universalité des droits de l'homme. Les ONG biomédicales / humanitaires d'aujourd'hui se caractérisent par la diversité de leurs domaines et de leurs lieux d'intervention, dont le développement durable, la lutte contre la famine, contre la maladie, contre les épidémies, le secours d'urgence en cas de catastrophes naturelles, d'inondations et de séismes, de conflits armés internes ou internationaux, la lutte contre le sida, la sensibilisation et l'éducation au développement. C'est à cet ensemble de tâches auxquelles s'adonne aussi MSF, I'une des ONG les plus puissantes et les plus actives sur le marché de l'humanitaire contemporain. À l'âge de l'humanitaire, au nom de l'universalité des droits de l'homme et du droit d'ingérence consécutif, l'aide n'est plus un acte concret, mais un devoir abstrait, dont les institutions internationales du village planétaire sont les vecteurs technocratiques. Les ONG biomédicales prennent l'avant-scène du développement au nom du droit de l'homme à la santé (Ferré, 1995 ; Lecherry et Ryfman, 1993). L'industrie du développement, devenue celle de l'aide humanitaire, procure aujourd'hui une infrastructure transnationale pour accueillir les «missionnaires » en toutes parts du monde.

\section{" Tourisme humanitaire " et recherche de bien-être}

Selon Chambers, I'un des arguments majeurs utilisés pour populariser le tourisme moderne est qu'il est bon pour nous. Pour mieux apprécier l'association loisir / travail dans le cas du tourisme humanitaire, «[...] we might keep in mind that the word travel derives from the Middle English word travail, which is associated with difficult, onerous and torturous realms of experience " (Chambers, 2000 : 16) ; le tourisme contient déjà en soi un élément de travail, selon cette conception. Voilà précisément ce que peut rechercher le chirurgien qui optera pour une pratique intensive en Afrique pour ses deux semaines de vacances, à savoir un métissage loisir / travail. Que ce soit le désillusionnement d'un poste de travail entre quatre murs d'hôpital, la volonté d'aider quelqu'un d'autre ou encore le désir de se sentir utile (de sauver des vies, par exemple) qui motive à l'action, l'attrait touristique de découvrir de nouveaux peuples et de nouvelles civilisations plane toujours derrière un engagement dans l'aide humanitaire. L'aide humanitaire internationale mène hors des sentiers battus, vers un rapport à l'Autre intense et souvent flatteur (à titre de sauveteur); il procure un soutien loin de chez soi (un réseau convivial pour de nombreux drifters) ; il offre une expérience hors de l'ordinaire, une expérience exotique, dépaysante, un défi professionnel en même temps

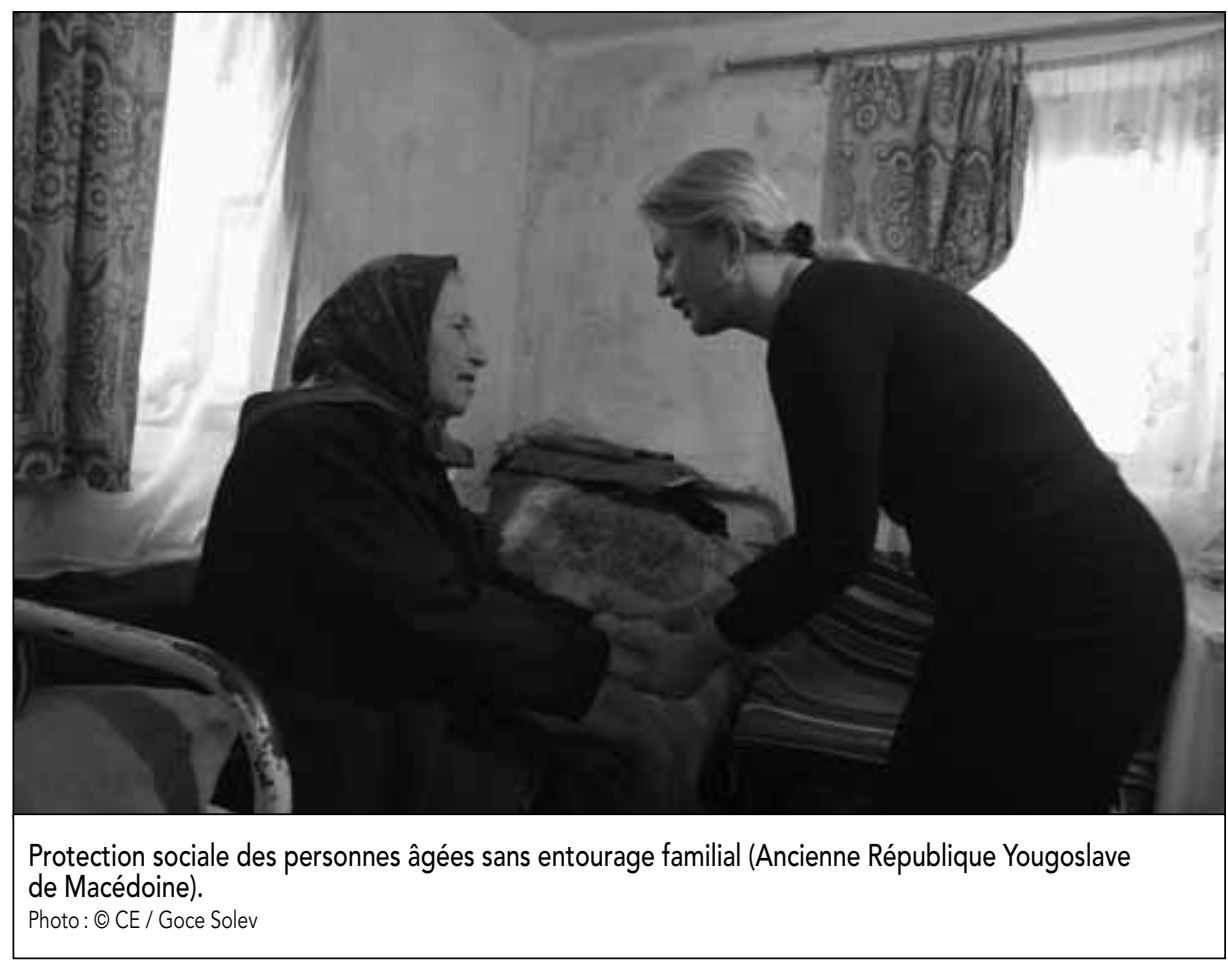

Protection sociale des personnes âgées sans entourage familial (Ancienne République Yougoslave de Macédoine).

Photo: : CE / Goce Solev

qu'un geste moral, éthique. II consiste en une combinaison agrément / travail, un métissage devant équilibrer les bienfaits pour soi et pour les autres chez qui l'on séjourne.

Le plaisir initial du travail en lieux exotiques s'estompe évidemment rapidement; pour les initiés, l'aspect voyager ou connaître des nouveautés n'existe quasiment plus, surtout pour des personnes qui ont fait de l'aide humanitaire toute leur vie et pour qui c'est l'engagement dans le travail qui prime. Le degré de motivation pour une recherche loisir/ travail assure les distinctions à apporter. L'intensité de l'expérience des interactions avec l'Autre qui s'engage dans une atmosphère de survie, de travail, de solidarité et de moralité peut certes être une composante d'un voyage pour son plaisir propre. Quand l'aspect voyage, conquête de nouveautés et exploration n'existe plus, il n'est plus tellement question de tourisme.

L'aide humanitaire, en fonction de la nature de l'expérience qu'elle offre et en fonction de l'impression qu'elle engendre de faire quelque chose d'incontestablement «bon » pour soi et pour l'Autre, peut définitivement procurer un bien-être initial pour le touriste en quête de sensations fortes et dépaysantes. Dans un second temps, le «normal » peut s'installer et l'intensité des expériences humanitaires, les longs déplacements loin 
des siens, la vie en communauté dans des conditions extrêmes, les situations traumatisantes de la guerre, des catastrophes naturelles ou sociales peuvent à la longue contrer et fragiliser l'équilibre du bien-être initial acquis. L'infrastructure de certaines organisations humanitaires telles que Médecins Sans Frontières avec laquelle je suis plus familière ${ }^{9}$, par exemple, utilise implicitement l'aspect "tourisme » de l'aide humanitaire comme outil thérapeutique avec ses intervenants sur le terrain; peut-être pour renouveler les bienfaits de l'expérience initiale du dépaysement. La rotation des équipes de travail, les déplacements vers de nouvelles cultures et des nouveaux paysages rencontrent les besoins de nouveauté, d'exploration du touriste humanitaire et contribuent souvent à restituer / rétablir le bien-être personnel recherché dans le voyage. II s'agit là d'une stratégie que certains ont perçue clairement : "quand ils me voient comme ça (un peu triste et dépressif), MSF me déplace simplement vers de nouvelles villes et contrées " avoue un médecin argentin de MSF-OCBA (centre opérationnel BarceloneAthènes) lors d'une communication personnelle (mars 2005).

Dans le sens inverse, cependant, l'ONG, ici MSF, offre de constants retours vers la culture de l'organisation, cherchant ainsi à soutenir ses équipes sur le terrain, mais maintenant par la même occasion un certain contrôle sur le degré d'intégration à l'intérieur de la culture locale. Des rencontres, des formations, des cours, des briefings, des allers-retours constants des centres opérationnels aux projets, empêchent la dérive des volontaires vers la culture de l'hôte. Une telle façon de faire contrecarre peut-être la possibilité pour la culture locale de pénétrer le projet dont elle est pourtant la pièce centrale.

On peut percevoir le tourisme humanitaire comme une forme de nomadisme qui concilie initialement travail et loisir ; l'expérience sur le terrain suscite une intensité au travail qui peut se comprendre par le fait d'être loin de chez soi pendant de longues périodes de temps, le plus souvent dans des situations précaires, conflictuelles et intenses d'un point de vue humain. Le côté loisir se recherche et se retrouve dans la convivialité, dans les festivités intenses et coutumières entre les membres de l'organisation, presque tous des célibataires âgés de 25 à 45 ans. Lors du cours de pré- paration au premier départ, les nouveaux «missionnaires» se présentent devant le groupe en mentionnant tous, à quelques exceptions près, leur état civil ; les séparations récentes et le célibat constituent définitivement la norme. La quête de sortir de son quotidien, peut-être de fuir une situation inconfortable (ou trop confortable), d'entrer en interaction avec de nouvelles personnes de culture et de civilisation différentes est fortement recherchée et imprègne le parachutage dans des coutumes locales étrangères. Le goût intense pour de nouvelles expériences culturelles, souvent "extrêmes ", des expériences limites (aux limites des interdits collectifs locaux et transnationaux) se greffe communément, bien qu'officieusement et à ses propres risques, à l'aide humanitaire.

Le duo puritanisme-décadence semble aller de pair. On peut se rappeler que les « débauches touristiques se sont développées alors que le puritanisme se maintenait au sommet de son pouvoir dans la société anglaise des années 1750-1800" (Laplante 1999 : 20). Un désir d'améliorer la condition humaine et un désir de sensations extrêmes aux limites de l'interdit et de ses fruits défendus ne sont pas aussi contradictoires qu'ils ne le laissent paraitre ; l'un et l'autre remettent en question la situation actuelle tout en y cherchant une alternative plus viable. Qu'il soit compensatoire des désillusions puritaines à voir la décadence humaine lors de situations de guerre et de conflit, ou qu'il soit simplement une manière d'accepter des pratiques culturelles de certains pays qui possèdent des mœurs autres, le plaisir éphémère du tourisme extrême (sexuel, expérimental des drogues locales, hors des limites des sentiers battus...) «hante " en quelque sorte l'aide humanitaire, tant de l'intérieur que de l'extérieur. Les pratiques de tourisme extrême chez les intervenants de MSF sont tout à fait classiques; elles longent les marges de l'interdit et les dépassent fréquemment. La position de l'organisation MSF face au tourisme extrême est très libérale et fonctionne sous le seuil de la tolérance. Un code de conduite existe et il est en pleine discussion, à savoir s'il doit être renforcé. MSF se trouve aux prises avec des problèmes liés à son double statut d'agence humanitaire dotée d'un rôle d'autorité, mais aussi de tourisme humanitaire exposé aux écarts de la recherche du bien-être personnel.
Un scandale a éclaté tout récemment dans les médias suédois concernant la position ou non-position que MSF prenait vis-à-vis du tourisme sexuel et des drogues douces. Une médecin suédoise a refusé de devenir volontaire MSF parce qu'elle n'était pas d'accord avec la non-politique de I'ONG concernant le tourisme sexuel. En Suède, la prostitution est illégale. Les missionnaires de la Croix-Rouge et de l'Agence canadienne de développement international (ACDI) doivent signer un document qui signale clairement ce qui est considéré comme abus de pouvoir, surtout en matière de prostitution. MSF demeure plus libérale à cet égard et affirme que l'organisation n'est pas contre la prostitution telle quelle (ni contre les drogues douces), mais des visites avec des prostitués sont prohibées dans les véhicules et les prémisses de MSF et, surtout, sont interdites avec des personnes de la population ciblée par l'intervention. Le tourisme sexuel est à cet effet laissé à la discrétion de chacun, une forme de respect pour les libertés individuelles, tant qu'elles ne menacent pas d'outrage devant les interdits collectifs de la communauté internationale.

Le tourisme sexuel soulève une polémique surtout au sein des centres opérationnels internationaux qui doivent assumer la responsabilité de leur philanthropie humanitaire. "MSF a un rôle particulièrement clé dans la lutte contre le sida et il devient contradictoire à leur cause lorsqu'à la même instance elle accepte le fait que leurs missionnaires (expatriés) voient des prostituées » (Johan Schaar, Directeur au département de l'aide humanitaire de l'ACDI ${ }^{10}$ ). Le tourisme sexuel est déjà condamnable alors qu'il entre en conflit avec les mesures préventives du sida et les principes de santé publique promulgés, mais il est d'autant plus condamnable alors qu'il soulève des questions de possibilité d'abus de pouvoir. "C'est déjà assez mal quand un touriste achète des services sexuels, mais c'est encore pire quand un individu représentant une organisation d'aide le fait » (Pehr-olov Pehrson, médecin, président section suédoise de $\mathrm{MSF}^{11}$ ), peut-être parce que le fruit interdit lui est encore plus interdit qu'aux autres. Les enjeux de pouvoir sont énormes entre un expatrié et la population qu'il traite, engage et côtoie. On appelle alors à un tourisme modéré qui ne ternira pas l'image internationale de l'aide humanitaire. 


\section{Tourisme et rapport à l'Autre (ou l'hôte)}

Le tourisme humanitaire imprègne des relations de pouvoir avec les résidants des pays-hôtes, qui ne sont pas tout à fait conformes aux visions humanitaires; de par son statut d'appartenance à des pays étrangers dits avancés et civilisés, ses alliances capitalistes, scientifiques et technologiques et son intervention en situation d'autorité au-

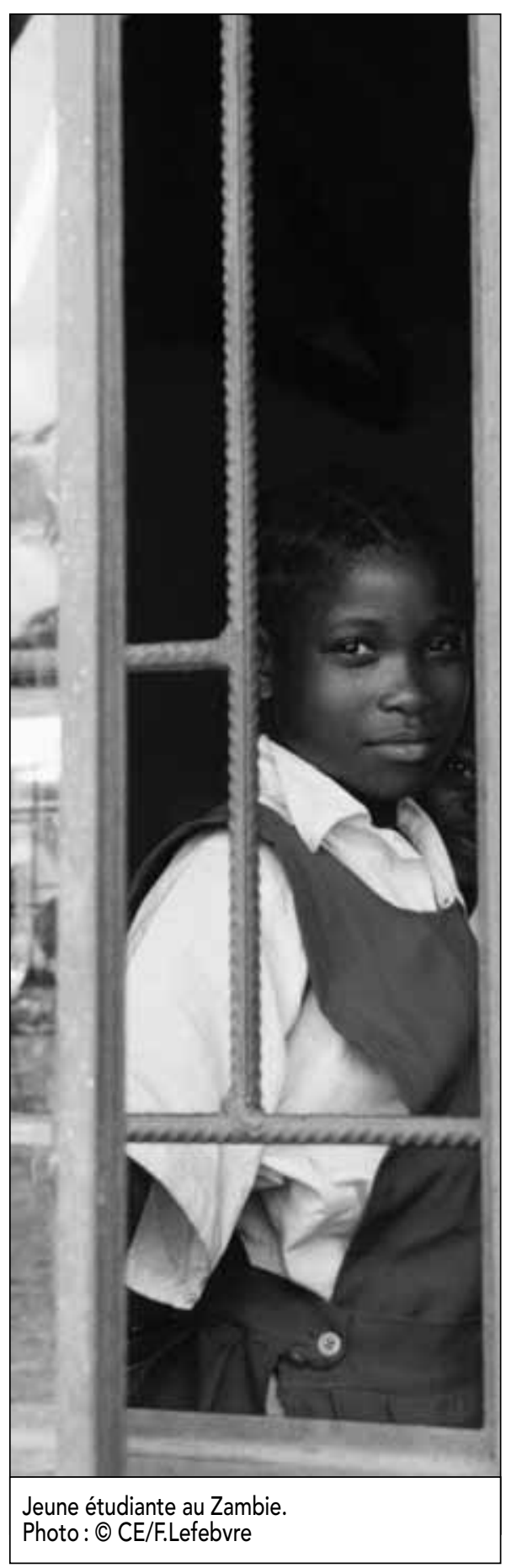

près de populations démunies, il incite aux abus de pouvoir. L'infrastructure de MSF, par exemple, procure au coordonnateur de projet basé dans une ville du pays-hôte, selon certains points de repères sur le comportement (Blackburn 2005), l'entière disposition du sort de son personnel local et l'espace pour « inventer », en quelque sorte, les politiques de conduite à l'intérieur de l'équipe. Les interventions visant à rendre accessibles la santé et le bien-être aux populations démunies en tant que droit humain ont non seulement un caractère utopique, mais elles sont "euro-centriques", c'est-à-dire qu'elles reflètent essentiellement des idéaux européens. Plusieurs personnes des pays en développement se joignent aujourd'hui aux équipes de MSF et peuvent partager les mêmes idéaux. II demeure que la manière proposée d'obtenir la santé et le bien-être pour tous aurait pu avoir lieu autrement s'il y avait une plus grande marge de manœuvre. II y a néanmoins généralement peu de place allouée aux savoirs thérapeutiques et aux idéaux locaux. De plus, le personnel local impliqué dans l'aide humanitaire n'a pas accès aux mêmes libertés individuelles telles que définies par la communauté internationale.

Le tourisme humanitaire génère dans le domaine du tourisme un nouveau rapport à l'Autre et à la culture de l'Autre, un rapport plus intime et plus personnalisé, mais parfois aussi une interaction de dominant-dominé difficile à démanteler. II procure un certain bien-être à ses volontaires par le biais des déplacements vers de nouvelles cultures, d'une interaction avec les personnes de ces cultures et l'impression, du moins initiale, de participer au bien-être physique de ces populations. La philanthropie humanitaire réclame les mêmes droits pour tous, tel que le prône le Code mondial d'éthique du tourisme ${ }^{12}$, mais le tourisme qui se rapporte à l'aide humanitaire demeure la donne de rares privilégiés, ce qui explique en partie les tentatives de plus en plus nombreuses d'impliquer les homologues locaux non seulement sur le plan local des projets, mais encore à l'intérieur du réseau international.

Les courants de participation communautaire, d'implication d'homologues locaux, appellent un dialogue accru entre les cultures, mais le plus souvent ce dialogue est confiné à l'intérieur d'une dynamique de victime-sauveteur, le sauveteur tirant sa satisfaction de son rôle qui est de transmettre son expertise et sa technique à l'Autre. « Nul besoin de preuves pour soutenir l'affirmation selon laquelle le tourisme peut être le meilleur ami aussi bien que le pire ennemi du développement » (Unesco, 2005).

Comme la révolution verte et biologique des années 1960, la révolution de l'humanitaire qui alimente celle du tourisme engendre de nouvelles dépendances. Les libertés individuelles et les interdits collectifs que dicte la communauté internationale sont imprégnés de valeurs culturelles à essence occidentale; ces valeurs impliquent entre autres que le bien-être physique, mental et social dépend de savoirs et de techniques spécifiques, les savoirs biomédicaux et leurs biotechnologies actuellement en tête des besoins jugés «essentiels ». Les ONG du tourisme se joindront peut-être à l'avant-scène du développement au nom du droit de l'homme à la liberté des déplacements touristiques. II s'agit que le bien-être devienne plus étroitement lié au dépaysement essentiel qu'aux traitements biologiques des corps, soit que le bien-être redevienne associé à l'âme, cette fois une âme qui s'épanouit en accédant directement et personnellement aux merveilles de la planète. Cela implique encore le transfert de connaissances et de techniques et, surtout, la définition de certaines manières de conduire ces échanges et certaines manières de se conduire lors de ces échanges.

Le tourisme humanitaire n'est plus seulement redevable aux libertés individuelles, mais il est de plus en plus restreint par des interdits collectifs. II se veut aujourd'hui un tourisme modéré qui se doit d'être attentif à sa propre culture ainsi qu'à la culture de l'Autre, telle que définie par le marché humanitaire. C'est dans de nouvelles formes de rapport à l'Autre qu'évolue le tourisme humanitaire, un rapport à l'Autre qui est défini par la communauté internationale, par les organisations qui facilitent lesdits rapports et par ceux qui interagissent à l'intérieur de ces rapports. Le rapport à l'Autre dans le tourisme humanitaire a donc fluctué du simple rapport dont la satisfaction est d'abord recherchée pour soi vers un rapport loisir / travail davantage dirigé vers la satisfaction de l'Autre en même temps que vers la satisfaction et le plaisir personnels. Conséquemment, le rapport à l'Autre s'établit dans une plus grande proximité, dans un objectif commun du bien-être pour tous, mais tel que défini par la communauté internationale. 


\section{Perspectives: dynamique du tourisme}

Le bien-être pour tous tel que défini par les membres de la communauté internationale et la dynamique de transferts de connaissances techniques humanitaires et touristiques telle que vécue au sein des organisations humanitaires non gouvernementales constituent, il va s'en dire, des barrières au dialogue entre les cultures et les civilisations. Les cultures " authentiques » non capitalistes constituent un attrait touristique pour les Occidentaux en même temps qu'elles deviennent les cibles privilégiées de l'aide humanitaire ; émergent alors des échanges culturels inégaux dans lesquels les techniques des pays industrialisés plus riches se définissent comme outils indispensables du bien-être des pays plus démunis. On alloue peu de temps et d'espace pour partager et apprendre ensemble d'autres formes de bien-être que celles adoptées par les sociétés occidentales ; le dialogue demeure unidirectionnel plutôt qu'équitable et multidirectionnel.

Les visées altruistes du tourisme humanitaire, mondial, équitable, responsable et durable ne constituent plus un tourisme « libre » sans toits ni lois. L'action humanitaire est largement gouvernée par les valeurs exportées par les réseaux transnationaux, que ce soit celles de l'éthique médicale universelle, celles du Code mondial d'éthique du tourisme: la gouvernance des comportements, la surveillance des sociétés dites développées s'étend à toutes parts du monde.

Julie Laplante, auteure de Pouvoir Guérir. Médecines autochtones et humanitaires (2004), est post-doctorante en Anthropologie à l'Université de Montréal (FCAR et CANVAC).

\section{Notes}

1 «Le tourisme social est né d'une exigence éthique portée par une revendication militante dont la légitimité repose sur l'affirmation d'un droit, le droit au tourisme, qui constitue le prolongement naturel du droit au travail et de son corollaire, le droit au repos, aux congés payés et donc aux vacances. » (Bélanger, 1999: 53)

2 Résolution adoptée par l'assemblée générale des Nations unies le 21 décembre 2001, [http://www.world-tourism.org/code_ethics/ fr/brochure.htm].
3 [http://www.tourisme.equipement.gouv.fr/fr/ navd/dossiers/tour_ethiqe/charte_france.jsp], consulté le 30 octobre 2005.

4 Cette section est une version remaniée du chapitre 3 dans Laplante (2004).

5 Le Comité d'Oxford de lutte contre la famine, se donnant pour objectif de secourir les populations civiles grecques affamées par l'occupant nazi (aujourd'hui l'OXFAM), se constituait ainsi en 1942. Le mouvement a eu un essor encore plus grand aux États-Unis avec le Catholic Relief Service (CRS) et le Cooperative for American Relief in Europe (CARE), qui résulte de 23 organisations. Enfin, c'est dans cette même filiation universaliste que s'inscrivent les ONG axées sur la défense et la promotion des droits de l'homme, telle Amnistie Internationale.

6 Voir la revue Économie et humanisme et les écrits du père Lebret dans Hours (1998: 100-101).

7 [http://www.msf.ca/ausujetde/charte.htm].

8 L'organisation Environnement et développement du tiers-monde (ENDA), créée en 1972, peut fournir un exemple à cet effet. Son siège est à Dakar. Elle a surtout été active en Afrique, mais son action est aujourd'hui quasi planétaire puisqu'elle est aussi présente en Asie (Inde, Thaillande) et en Amérique latine (Brésil, Colombie, Caraïbes...).

9 Je côtoie cette ONG depuis 1992 à titre de bénévole, de chercheure-anthropologue, de volontaire et de logisticienne.

10 Publié dans un journal suédois, Göteborgsposten, le 11 septembre 2004.

11 Publié dans Göteborgsposten, le 29 août 2004.

12 [http://www.world-tourism.org/code_ethics/ $\mathrm{fr} /$ brochure.htm

\section{Bibliographie}

Bélanger, Charles-Étienne (1999), «Le tourisme social : Bilans, enjeux et perspectives ", Téoros, vol. $18, n^{\circ} 3$, p. 53-57.

Blackburn, Philippe (2005), "Comportement chez MSFCH. Recommandations finales ", Rapport MSF, Genève, 4 août.

Burch, S., et O. León (1996), "Amérique latine: La biopiraterie dans les relations Nord-Sud ", [http://www.globenet.org/horizon-local/dial/ 2135.html], consulté le 27 juin 2003. Article paru dans ALAl, Équateur, 27 juin.

Cazes, Georges (1999), «Tourisme et relations internationales: Perspective cavalière du dernier demi-siècle ", Téoros, vol. 18, n 3, p. 4-10.

Chambers, Erve (2000), Native Tours: The Anthropology of Travel and Tourism, Illinois, Waveland Press.

Cohen, Erik (2004), Contemporary Tourism: Diversity and Change, Amsterdam, Elsevier.
Escobar, Arturo (1997), «Anthropologie et développement », Revue internationale des sciences sociales, décembre, vol.154, p. 539-560.

Escobar, Arturo (1995), Encountering Development: The Making and Unmaking of the Third World, Princeton University Press.

Escobar, Arturo (1983), Discourse and Power: An Historical Perspective on the Formation of Development Theory and Practice (19451955), UNESCO SS-83/CONF. 618/15, novembre, Paris.

Escobar, Arturo (1984-1985), «Discourse and Power in Development: Michel Foucault and the Relevance of His Work to the Third World ", Alternatives, vol. X, p. 377-400.

Ferré, J.L. (1995), L'action humanitaire, Toulouse, Édition Milan.

Hours, Bernard (1998), L'idéologie humanitaire ou le spectacle de l'altérité perdue, Paris, L'Harmattan.

Laplante, Julie (2003), «Le médicament aux frontières des savoirs humanitaires et autochtones. ", Anthropologie et sociétés. vol. 27, $\mathrm{n}^{\circ} 2$, p. 59-75.

Laplante, Julie (2004), Pouvoir guérir. Médecines autochtones et humanitaires, Québec, Presses de l'Université Laval.

Laplante, Marc (1999), "L'expérience touristique dans une société nomade: Quel avenir?", Téoros, vol. 18, n³, p. 17-21.

Lecherry, C., et P. Ryfman (1993), Action humanitaire et solidarité internationale: les ONG, Paris, Hatier.

Morisset, Lucie K., et Alain Caron (1999), "Le XIXe siècle, cent ans plus tard: Quelques notes pour repenser les rapports tourisme/culture/identité ", Téoros, vol. 18, n³, p. 22-28.

Picard, Michel, et Robert E. Wood (1997), Tourism, Ethnicity, and the State in Asian and Pacific Societies, Honolulu, University of Hawaii Press.

Smith, Valene L. (1989, $2^{\mathrm{e}}$ édition, [1 ${ }^{\text {re }}$ édition 1977]), "Introduction », dans V. Smith (dir.), Hosts and Guests: The Anthropology of Tourism, Philadelphia, University of Pennsylvania Press, p. 1-17.

Unesco, Secteur de la culture (2005), [http:// portal.unesco.org/culture/fr/ev.php-URL_ID= 11408\&URL_DO=DO_TOPIC\&URL_SECTION $=201 \cdot \mathrm{html}$.

Urbain, Jean-Didier (1994), Sur la plage. Mceurs et coutumes balnéaires, Paris, Plon et Rivages. 